\title{
COLDFIB - The New FIB Source from Laser Cooled Atoms
}

\author{
A. Delobbe ${ }^{1}$, M.Reveillard ${ }^{1}$, M. Viteau ${ }^{1}$, A. Houel ${ }^{1}$, and D.Comparat ${ }^{2}$ \\ 1. Orsay Physics, Tescan Orsay Holding, 13710 Fuveau, France \\ 2. Laboratoire Aimé Cotton, Université Paris-Sud, ENS Cachan, CNRS, Université Paris-Saclay, 91405 \\ Orsay Cedex, France
}

Charged particle beams of controlled energy and strong focusing are widely used tools in industry and academic, as a Focused Ion Beam (FIB) column combined with a Scanning Electron Microscope (SEM) provide full control of nanofabrication or nanolithography processes. Ion energy can be varied typically in the 1-30 keV range, with an energy-dependent resolution reaching tens of nanometer range. Today, state-of-the-art FIBs commercially available are based mainly on plasma, liquid metal tips or Gas Field Ion Sources (GFIS) for respectively large, intermediate, and low currents.

Despite the very high technological level of the available machines, research on new ion sources allowing even higher resolutions and a wider choice of atomic or molecular ions for new and demanding applications is very active. For example, the world of electronics components evolves regularly towards the miniaturization by integrating a larger number of transistors on a given area. As the dimensions become smaller $(10 \mathrm{~nm}, 7 \mathrm{~nm}$, and even $5 \mathrm{~nm}$ technology), the traditional instruments of analysis, such as the conventional FIB, reach their limits. It is today necessary to realize a technological breakthrough to be able to observe, analyze and modify components and structures at the nanometer scale.

Our new system, COLDFIB, takes up this nano-manufacturing challenge by coupling two advanced technologies: the laser cooling of atoms, and the manipulation of charged particles. This very innovative industrial solution, based on a source of $\mathrm{Cs}^{+}$ions obtained from laser cooled and ionized atoms, will allow to obtain ions beam featuring unequalled performances, and allow the milling of a few nanometers large patterns. This method creates an atoms jet and then ions beam with very small angular divergence and energy spread. This allows for extremely good performances at every beam energy. This new technology offers a resolution 10 times higher than the one obtained with LMIS (at $5 \mathrm{keV}$ ), and reaches the nanometer at $30 \mathrm{keV}$ (Figure 1).

Here we will present the first installation of this kind of instrument on a SEM-FIB TESCAN microscope. The principle of atom cooling, Rydberg excitation and ionization will be explained [1], as well as expected performances [2]. In particular, the integration of the lasers and the Cs oven into the microscope will be demonstrated.

The main results in terms of image resolution at different currents and energies will be discussed. We will focus on the 2 main applications which are SIMS analysis and circuit edit. In fact, it appears clearly that SIMS analysis could benefit from a medium energy range (3-10 keV) Cs beam offering high resolutions. COLDFIB could bring a real technological leap in comparison with existing Cs sources.

Moreover, low energies offered by cold atoms are a real advantage both for circuit edit and low damage milling. In this study, this will be illustrated through several milling examples, at various energies and currents (from several nA down to less than $1 \mathrm{pA}$ ).

As a conclusion, we will compare the different sources options for FIB : Ga source, Xe Plasma source, He Gas field Ion Source and cold atoms in terms of ultimate resolution, energy range and current range. 
Those features will be connected with expected applications, and we will present COLDFIB as a new ion source, answering the future challenges of high resolution, low energy and local ion milling.

References :

[1] L. Kime et al, Phys. Rev. A 88 (2013) 033424

[2] M. Viteau et al, Ultramicroscopy 164 (2016) p. 70-77
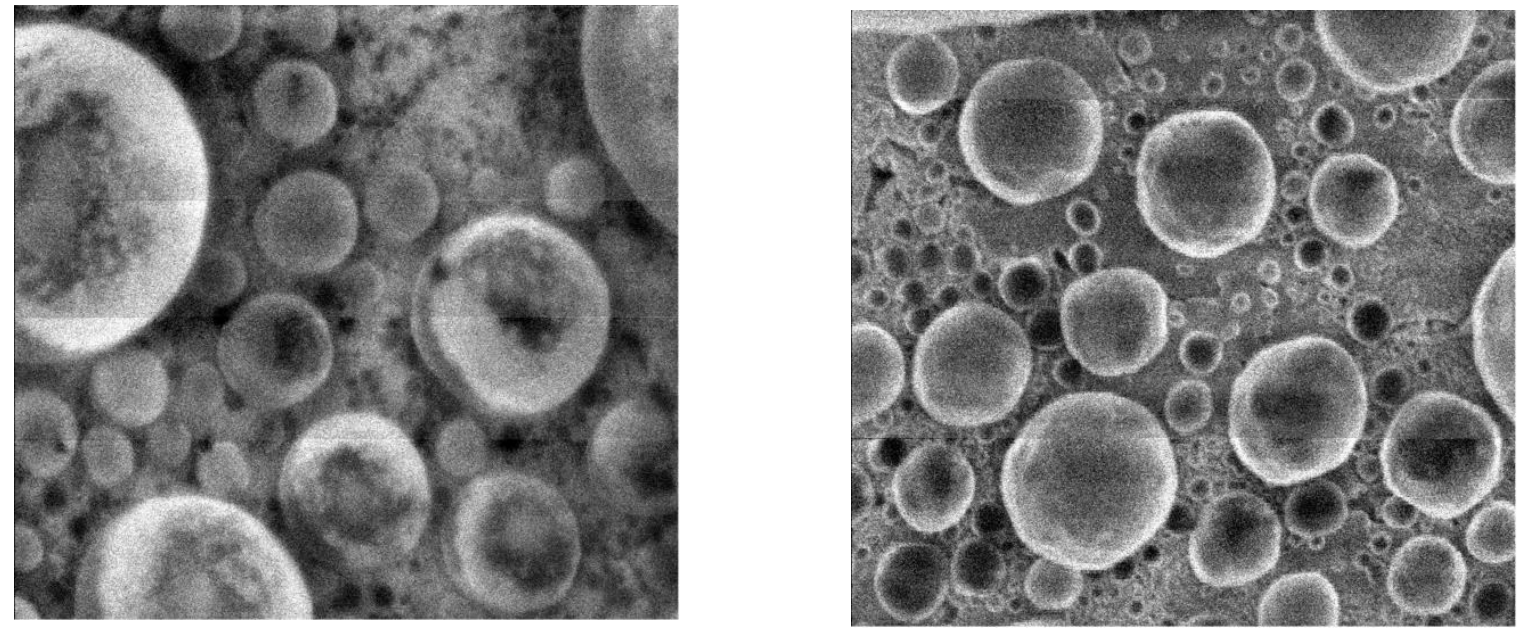

Figure 1. Tin on carbon at $5 \mathrm{keV}$ and $30 \mathrm{keV}$ (Field of view of 10 and $5 \mu \mathrm{m}$ respectively).
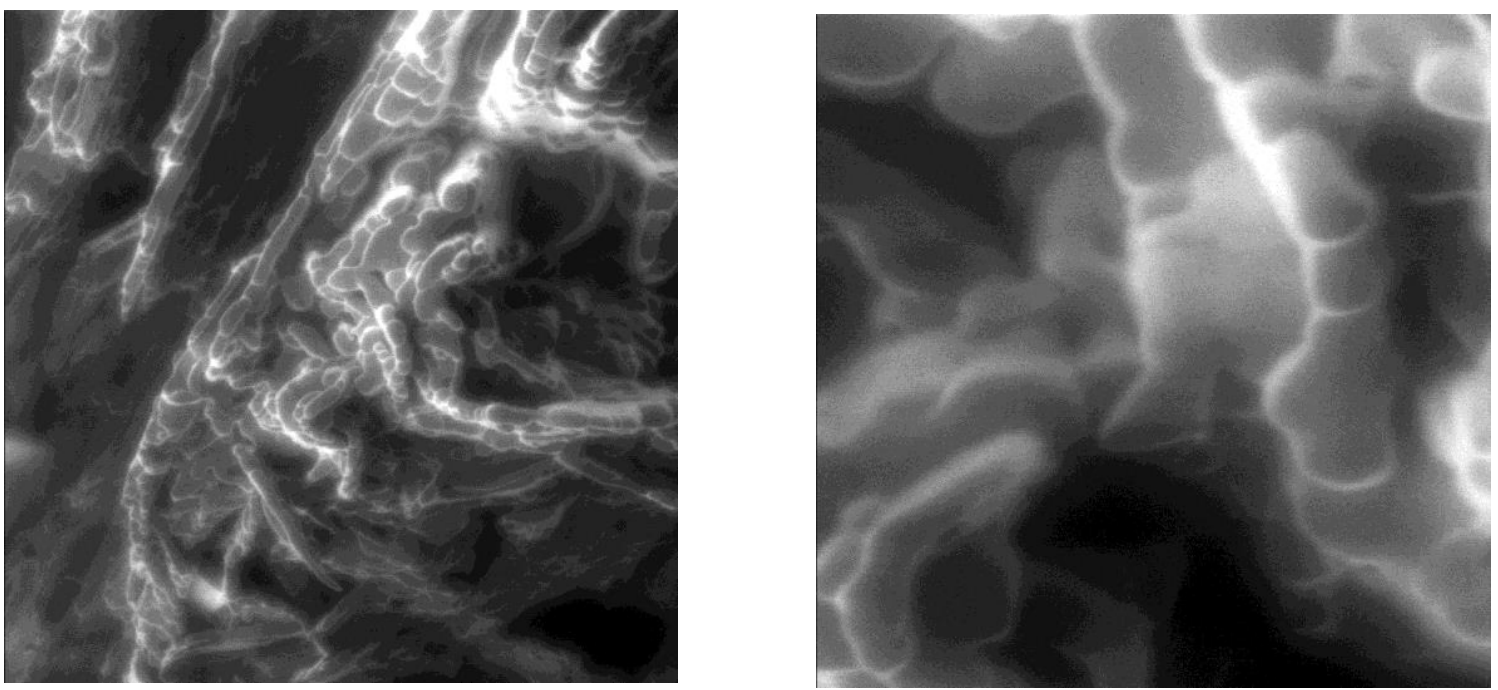

Figure 2. Carbon graphite : images at $30 \mathrm{keV}$ (Field of view of 5 and $1 \mu \mathrm{m}$ respectively). 